\title{
A MODIFIED LOG-HARNACK INEQUALITY AND ASYMPTOTICALLY STRONG FELLER PROPERTY
}

\author{
LIHU XU
}

\begin{abstract}
We introduce a new functional inequality, which is a modification of log-Harnack inequality established in 21 and [30, and prove that it implies the asymptotically strong Feller property (ASF). This inequality seems to generalize the criterion for ASF in [14, Proposition 3.12]. As a example, we show by an asymptotic coupling that $2 \mathrm{D}$ stochastic Navier-Stokes equation driven by highly degenerate but essentially elliptic noises satisfies our modified log-Harnack inequality.
\end{abstract}

AMS subject Classification: 60J75, 60J45.

Keywords: Wang type Harnack inequality, log-Harnack inequality, modified logHarnack inequality, strong Feller property, asymptotically strong Feller property, 2D stochastic Navier-Stokes equation, asymptotic coupling.

\section{INTRODUCTION}

Dimensional free Harnack inequality was introduced by Wang in [26] to study the diffusions on Riemannian manifolds (see also [3, 4, for further development). Wang type Harnack inequality has been applied to many research problems such as studying ultracontractivity and functional inequalities ([23, 22, 27, 28]), shorttime behaviors of infinite-dimensional diffusions ([1, 2, 17]), heat kernel estimates $([6,11])$ and so on. In recent years, this inequality has also been established and applied intensively in the study of SPDEs (see e.g. [23, 29, 19, 7, 9, 31, 33] and references within). Let $\left(P_{t}\right)_{t \geq 0}$ be a Markov semigroup on a Polish space $\mathcal{X}$, this type of Harnack inequality can be formulated as

$$
\left(P_{t} f\right)^{\alpha}(x) \leq\left(P_{t} f^{\alpha}\right)(y) \exp \left[C_{\alpha}(t, x, y)\right], \quad f \geq 0
$$

where $\alpha>1$ is a constant, $C_{\alpha}$ is a positive function on $(0, \infty) \times \mathcal{X}^{2}$ with $C_{\alpha}(t, x, x)=$ 0 , which is determined by the underlying stochastic equation.

Partial support by the European Research Council under the European Union's Seventh Framework Programme (FP7/2007-2013) / ERC grant agreement nr. 258237 is gratefully acknowledged. 
On the other hand, in some cases Wang type Harnack inequality is not available, so that the following weaker version (i.e. the log-Harnack inequality)

$$
P_{t} \log f(x) \leq \log P_{t} f(y)+C(t, x, y), \quad f \geq 1
$$

becomes an alternative tool in the study. In general, according to [30, Section 2], (1.2) is the limit version of (1.1) as $\alpha \rightarrow \infty$. This inequality has been established in [21] and [30], respectively, for semi-linear SPDEs with multiplicative noise and the Neumann semigroup on non-convex manifolds. [32] shows that stochastic Burgers equation driven by additive noises satisfies a log-Harnack inequality. As for the research on Wang type Harnack and log-Harnack inequalities on stochastic NavierStokes type equations, we refer to [33] and [32].

To our knowledge, nearly all the stochastic systems in the above literatures is forced by nondegenerate noises. It is natural to ask whether a stochastic system with degenerate noises satisfies Wang type Harnack or log-Harnack inequality. This seems still an open problem.

The aim of this paper is to study the case of the systems driven by highly degenerate noises. For highly degenerate systems, one is usually not able to prove the strong Feller property (see Example 3.15 of [14]). Since Harnack and log-Harnack inequality implies strong Feller property ([29], [21]), there is no hope to prove these inequalities for highly degenerate systems. On the other hand, many dissipative systems such as 2D Navier-Stokes and reaction-diffusion equations driven by highly degenerate noises $([14,15])$ have asymptotically strong Feller property, it is natural to ask whether we can establish a functional inequalities which implies the asymptotically strong Feller property. This is the main motivation that we introduce the modified log-Harnack inequality, which seems to give a criterion for asymptotically strong Feller property more general than that in [14, Proposition 3.12]. This inequality also gives some pointwise information on Markov semigroups.

Definition 1.1 (Modified log-Harnack inequality). Let $\left\{P_{t}\right\}_{t \geq 0}$ be a Markov semigroup on a Polish space $\mathcal{X}$, it satisfies a modified log-Harnack inequality if there exist some constants $\alpha>0, \beta \geq 0, C=C(|x|,|y|)>0, \tilde{C}=\tilde{C}(|x|,|y|)>0$ and a function $\delta(t) \geq 0$ with $\lim _{t \rightarrow \infty} \delta(t)=0$ such that

$$
P_{t} \log f(y) \leq \log P_{t} f(x)+C|x-y|^{\alpha}+\delta(t) \tilde{C}|x-y|^{\beta}|| D \log f \|_{\infty}
$$

for any bounded differentiable function $f \geq 1$ and $x, y \in \mathcal{X}$. Moreover, $C$ and $\tilde{C}$ are both continuous w.r.t. $|x|$ and $|y|$.

The main results of this paper are the following Theorem 1.2, Corollary 1.4 and Theorem 1.5. The first two will be proven in section 2, while the last one will be shown in section 3 ,

Theorem 1.2. If a Markov semigroup satisfies a modified log-Harnack inequality, then it is asymptotically strong Feller. 
Hairer and Mattingly gave a criterion for asymptotically strong Feller property as the following.

Proposition 1.3 (Proposition 3.12 in [14]). Let $\left\{P_{t}\right\}_{t \geq 0}$ be a Markov semigroup on $\mathcal{X}$. If $\left\{P_{t}\right\}_{t \geq 0}$ satisfies the following inequality

$$
\left|D P_{t} f(x)\right| \leq C\left(\|f\|_{\infty}+\delta(t)\|D f\|_{\infty}\right)
$$

for any bounded differentiable function $f: \mathcal{X} \rightarrow \mathbb{R}$, where $C=C(|x|)>0$ and $\delta(t) \geq 0$ with $\lim _{t \rightarrow \infty} \delta(t)=0$, then $\left\{P_{t}\right\}_{t \geq 0}$ is asymptotically strong Feller.

The next corollary claims that (1.3) with $\alpha=2$ and $\beta=1$ implies a gradient estimate similar to (1.4). Therefore, the modified log-Harnack inequality, in some sense, seems to give a more general criterion for asymptotically strong Feller property. Moreover, (1.3) also provides some pointwise information of the semigroups.

Corollary 1.4. If a Markov semigroup $\left\{P_{t}\right\}_{t \geq 0}$ satisfies (1.3) with $\alpha=2$ and $\beta=1$, then

$$
\left|D P_{t} f(x)\right| \leq\|f\|_{\infty}^{2}+C+\delta(t) \tilde{C}\|D f\|_{\infty}
$$

for any bounded differentiable function $f$, where $C=C(|x|)$ and $\tilde{C}=\tilde{C}(|x|)$ and $\lim _{t \rightarrow \infty} \delta(t)=0$.

The following theorem claims that 2D stochastic Navier-Stokes equation forced by degenerate noises satisfies our modified log-Harnack inequality. We need to emphasize that those degenerate noises have essential ellipticity effect, see section 4.5 in 14 for more details.

Theorem 1.5. Let $\left\{P_{t}\right\}_{t \geq 0}$ be the Markov semigroup generated by Eq. (3.2) in section 3 below, a $2 D$ stochastic Navier-Stokes system forced by highly degenerate noises, then $\left\{P_{t}\right\}_{t \geq 0}$ satisfies a modified log-Harnack inequality, which has the exact form as in Theorem 3.3 below. Moreover, $\left\{P_{t}\right\}_{t \geq 0}$ satisfies (1.5).

Acknowledgements: We would like to gratefully thank Feng-Yu Wang for the stimulating and instructive discussions and carefully reading the first draft. We also would like to thank Jiang-Lun Wu for the encouragements. Part of the work was done during visiting Mathematics department of Swansea University.

\section{Modified LOG-Harnack InEQUAlity AND Asymptotically Strong FELLER PROPERTY}

Let us first recall the interesting conception of asymptotically strong Feller property, which was introduced by Hairer and Mattingly in [14]. For the more details, we refer to [14].

Definition 2.1. Let $\left\{d_{n}\right\}_{n}$ be an increasing sequence of pseudo metrics (pp 7 . [14]) on a Polish space $\mathcal{X}$. If $\lim _{n \rightarrow \infty} d_{n}(x, y)=1$ for all $x \neq y$, then $\left\{d_{n}\right\}$ is called a totally separating system of pseudo metrics for $\mathcal{X}$. 
Given a pseudo metric $d$, for any $d$-Lipschitz continuous function $f: \mathcal{X} \rightarrow \mathbb{R}$, we define the following semi-norm for $f$ :

$$
\|f\|_{d}=\sup _{\substack{x, y \in \mathcal{X} \\ x \neq y}} \frac{|f(x)-f(y)|}{d(x, y)} .
$$

Given $\mu_{1}$ and $\mu_{2}$, two positive finite Borel measures on $\mathcal{X}$ with equal mass, we denote by $\mathcal{C}\left(\mu_{1}, \mu_{2}\right)$ the set of positive measures on $\mathcal{X}^{2}$ with marginals $\mu_{1}$ and $\mu_{2}$, and define

$$
\left\|\mu_{1}-\mu_{2}\right\|_{d}=\inf _{\mu \in \mathcal{C}\left(\mu_{1}, \mu_{2}\right)} \int_{\mathcal{X}^{2}} d(x, y) \mu(d x, d y) .
$$

Definition 2.2 (Asymptotically strong Feller property). A Markov transition semigroup $P_{t}$ on a Polish space $\mathcal{X}$ is asymptotically strong Feller at $x$ if there exists a totally separating system of pseudo metrics $\left\{d_{n}\right\}_{n}$ for $\mathcal{X}$ and a sequence $t_{n}>0$ such that

$$
\inf _{U \in \mathcal{U}_{x}} \lim _{n \rightarrow \infty} \sup \sup _{y \in U}\left\|P_{t_{n}}(x, \cdot)-P_{t_{n}}(y, \cdot)\right\|_{d_{n}}=0,
$$

where $\mathcal{U}_{x}=\{U: U$ is the neighbourhood of $x\}$. We call that $P_{t}$ satisfies asymptotic strong Feller property if it is asymptotic strong Feller at each $x \in \mathcal{X}$.

Remark 2.3. If $\mathcal{X}$ has a metric, then the definition (2.1) is equivalent to

$$
\lim _{r \rightarrow 0} \lim _{n \rightarrow \infty} \sup \sup _{y \in B(x, r)}\left\|P_{t_{n}}(x, \cdot)-P_{t_{n}}(y, \cdot)\right\|_{d_{n}}=0,
$$

where $B(x, r)$ is the ball in $\mathcal{X}$ centered at $x$ with radius $r$ under this metric.

The following two lemmas are Lemma 3.3 and Corollary 3.5 of 14 respectively. The first one is a not difficult consequence of Monge-Kantorovich duality ([25]).

Lemma 2.4. Let $d$ be a continuous pseudo metric on a Polish space $\mathcal{X}$ and let $\mu_{1}$, $\mu_{2}$ be two positive measures on $\mathcal{X}$ with equal mass. Then we have

$$
\left\|\mu_{1}-\mu_{2}\right\|_{d}=\sup _{\|\varphi\|_{d}=1} \int_{\mathcal{X}} \varphi(x)\left(\mu_{1}-\mu_{2}\right)(d x) .
$$

Remark 2.5. By [25] (pp. 34), if $d$ is bounded we have

$$
\left\|\mu_{1}-\mu_{2}\right\|_{d}=\sup _{\substack{\|\varphi\|_{d}=1 \\\|\varphi\|_{\infty} \leq\|d\|_{\infty}}} \int_{\mathcal{X}} \varphi(x)\left(\mu_{1}-\mu_{2}\right)(d x) .
$$

Lemma 2.6. Let $\mathcal{X}$ be a Polish space and let $\left\{d_{n}\right\}$ be a totally separating system of pseudo metrics for $\mathcal{X}$. Then, $\left\|\mu_{1}-\mu_{2}\right\|_{T V}=\lim _{n \rightarrow \infty}\left\|\mu_{1}-\mu_{2}\right\|_{d_{n}}$ for any two positive measures $\mu_{1}$ and $\mu_{2}$ with equal mass on $\mathcal{X}$.

The following theorem is due to Hairer and Mattingly [14]. 
Theorem 2.7. If $P_{t}$ is an asymptotically strong Feller Markov semigroup and there exists a point $x$ such that $x \in \operatorname{supp}(\mu)$ for every invariant probability measure $\mu$ of $P_{t}$, then there exists at most one invariant probability measure for $P_{t}$.

Hairer and Mattingly proved by the above theorem, together with (1.4), the following important result: For 2D stochastic Navier-Stokes systems if at least two linearly independent Fourier modes with different Euclidean norms are driven, then the system is ergodic (see [14, Theorem 2.1] and the examples therein).

With the above quick review of asymptotic strong Feller property, we are now at the place to prove Theorem 1.2 and Corollary 1.4 .

Proof of Theorem 1.2. For any bounded differentiable function $f$, we choose some constant $\varepsilon>0$ small enough to make $\varepsilon\|f\|_{\infty}<1 / 2$. Applying the modified logHarnack inequality (1.3) to $2+2 \varepsilon f$, there exist some constants $\alpha>0, \beta \geq 0, C>0$, $\tilde{C}>0$ and some function $\delta(t) \geq 0$ with $\lim _{t \rightarrow \infty} \delta(t)=0$ such that

$$
\begin{aligned}
P_{t} \log (2+2 \varepsilon f)(y) \leq & \log P_{t}(2+2 \varepsilon f)(x)+C|x-y|^{\alpha} \\
& +\delta(t) \tilde{C}|x-y|^{\beta}|| D \log (2+2 \varepsilon f) \|_{\infty},
\end{aligned}
$$

which clearly implies

$$
\begin{aligned}
P_{t} \log (1+\varepsilon f)(y) \leq & \log \left(1+\varepsilon P_{t} f(x)\right)+C|x-y|^{\alpha} \\
& +2 \varepsilon \delta(t) \tilde{C}|x-y|^{\beta}\|D f\|_{\infty} .
\end{aligned}
$$

Since $\varepsilon\|f\|_{\infty}<1 / 2$, we have by Taylor expansion of the function $\log (1+x)$

$$
\varepsilon\left[P_{t} f(y)-P_{t} f(x)\right] \leq \varepsilon^{2}\|f\|_{\infty}^{2}+C|x-y|^{\alpha}+2 \varepsilon \delta(t) \tilde{C}|x-y|^{\beta}|| D f \|_{\infty} .
$$

Dividing by $\varepsilon$ on the both side of the above inequality and exchanging $x$ and $y$, one has

$$
\left|P_{t} f(y)-P_{t} f(x)\right| \leq \varepsilon|| f\left\|_{\infty}^{2}+\frac{C|x-y|^{\alpha}}{\varepsilon}+2 \delta(t) \tilde{C}|x-y|^{\beta}\right\| D f \|_{\infty}
$$

for any bounded differentiable $f$.

Next, we follow the idea in [14, Proposition 3.12]. For any $\gamma>0$, we define the metric $d_{\gamma}(x, y)=1 \wedge \frac{1}{\gamma}|x-y|$ for any $x, y \in \mathcal{X}$. It is clear that $\left\|d_{\gamma}\right\|_{\infty}:=$ $\sup _{x, y \in \mathcal{X}} d_{\gamma}(x, y) \leq 1$. For any $f$ differentiable function with $\|f\|_{d_{\gamma}} \leq 1$ and $\|f\|_{\infty} \leq\left\|d_{\gamma}\right\|_{\infty}$ (recall Remark 2.5), by $\|f\|_{d_{\gamma}} \leq 1$ one has $\|D f\|_{\infty} \leq \frac{1}{\gamma}$. (2.4) implies

$$
\left|\int_{\mathcal{X}} f(z) P_{t}(x, d z)-\int_{\mathcal{X}} f(z) P_{t}(y, d z)\right| \leq \varepsilon+\frac{C|x-y|^{\alpha}}{\varepsilon}+\frac{2 \delta(t) \tilde{C}|x-y|^{\beta}}{\gamma} .
$$


Since each bounded $f$ with $\|f\|_{d_{\gamma}}<\infty$ can be approximated by bounded differentiable function sequences, the above inequality and Lemma 2.4 implies

$$
\left\|P_{n}(x, \cdot)-P_{n}(y, \cdot)\right\|_{d_{\gamma}} \leq \varepsilon+\frac{C|x-y|^{\alpha}}{\varepsilon}+\frac{2 \delta(t) \tilde{C}|x-y|^{\beta}}{\gamma}
$$

Taking $\gamma=\sqrt{\delta(n)}$ and $\varepsilon=|x-y|^{\alpha / 2}$, we have

$$
\| P_{n}(x, \cdot)-\left.P_{n}(y, \cdot)\right|_{d_{\gamma_{n}}} \leq(1+C)|x-y|^{\frac{\alpha}{2}}+2 \sqrt{\delta(n)} \tilde{C}|x-y|^{\beta} .
$$

which, by (2.2), immediately implies that $\left(P_{t}\right)_{t \geq 0}$ is asymptotically strong Feller at $x$.

Proof of Corollary 1.4. From (2.4), taking $\varepsilon=|x-y|$ and letting $y \rightarrow x$, we immediately obtain (1.5).

\section{An example and Proof of Theorem 1.5}

In this section, we shall study 2D stochastic Navier-Stokes equation driven by highly degenerate but essentially elliptic noises as an example satisfying our modified log-Harnack inequality. 2D stochastic Navier-Stokes equation has been intensively studied in [14], 20], 8], [10], [18] and the references therein.

Let us first give a quick introduction to the background of 2D stochastic NavierStokes equations.

3.1. 2D stochastic Navier-Stokes systems. Let $\mathbb{T}^{2}=(\mathbb{R} / 2 \pi)^{2}$ and let

$$
\begin{aligned}
& L_{0}^{2}\left(\mathbb{T}^{2}, \mathbb{R}^{2}\right)=\left\{x \in L^{2}\left(\mathbb{T}^{2}, \mathbb{R}^{2}\right) ; \int_{\mathbb{T}^{2}} x(\xi) d \xi=0\right\}, \\
& H=\left\{x \in L_{0}^{2}\left(\mathbb{T}^{2}, \mathbb{R}^{2}\right) ; \operatorname{div} x=0\right\}
\end{aligned}
$$

Moreover,

$|\cdot|$ and $\langle\cdot, \cdot\rangle$ denote the norm and the inner product of $H$ respectively.

Let $\mathcal{P}: L_{0}^{2}\left(\mathbb{T}^{2}, \mathbb{R}^{2}\right) \rightarrow H$ be the orthogonal projection. Define the Stokes operator by

$$
A=\mathcal{P}(-\Delta)
$$

with $\Delta$ being the Laplacian on $L_{0}\left(\mathbb{T}^{2}, \mathbb{R}^{2}\right)$ and $D(A)=H^{2}\left(\mathbb{T}^{2}, \mathbb{R}^{2}\right) \cap H$. It is well known that $\left\{e_{k}=\frac{1}{2 \pi} e^{i k \cdot x}: k \in \mathbb{Z}^{2} \backslash\{0\}, x \in \mathbb{T}^{2}\right\}$ is an orthonormal basis of $L_{0}^{2}\left(\mathbb{T}^{2}, \mathbb{R}\right)$ and that $\Delta$ is self-adjoint with the spectrum $\left\{-|k|^{2}: k \in \mathbb{Z}^{2} \backslash\{0\}\right\}$. It is also clear that $\Delta e_{k}=-|k|^{2} e_{k}$. For any real number $\alpha$, one can define $(-\Delta)^{\alpha}$ by the spectral decomposition as

$$
(-\Delta)^{\alpha}=\sum_{k \in \mathbb{Z}^{2} \backslash\{0\}}|k|^{2 \alpha} e_{k} \otimes e_{k} .
$$


We can define the $\alpha$ order Stokes operator by

$$
A^{\alpha}=\mathcal{P}(-\Delta)^{\alpha}
$$

with the domain defined by (3.1) below.

Under the orthonormal basis $\left\{e_{k}\right\}_{k \in \mathbb{Z}^{2} \backslash\{0\}}, H$ can also be defined by

$$
H=\left\{x=\sum_{k \in \mathbb{Z}^{2} \backslash\{0\}} x_{k} e_{k}: x_{k} \in \mathbb{R}^{2}, k \cdot x_{k}=0, \sum_{k \in \mathbb{Z}^{2} \backslash\{0\}}\left|x_{k}\right|^{2}<\infty\right\} .
$$

Furthermore, we define

$$
D\left(A^{\alpha}\right)=\left\{x=\sum_{k \in \mathbb{Z}^{2} \backslash\{0\}} x_{k} e_{k}: x_{k} \in \mathbb{R}^{2}, k \cdot x_{k}=0, \sum_{k \in \mathbb{Z}^{2} \backslash\{0\}}|k|^{4 \alpha}\left|x_{k}\right|^{2}<\infty\right\} .
$$

It is clear that if $\alpha>0$ we have the following Poincare inequality

$$
|x| \leq\left|A^{\alpha} x\right|
$$

for any $x \in D\left(A^{\alpha}\right)$.

We shall study the following highly degenerate 2D stochastic Navier-Stokes type equation

$$
\left\{\begin{array}{l}
d X(t)+[\nu A X(t)+B(X(t), X(t))] d t=Q d W_{t} \\
X(0)=x
\end{array}\right.
$$

where

- $\nu>0$ is the viscosity constant.

- The nonlinear term $B$ is defined by

$$
B(u, v)=\mathcal{P}[(u \cdot \nabla) v], B(u)=B(u, u) \quad \forall u, v \in H^{1}\left(\mathbb{T}^{d}, \mathbb{R}^{d}\right) \cap H .
$$

- $W_{t}$ is the cylindrical Brownian motion on $H$ and $Q$ satisfies the highly degenerate condition as in Assumption 3.1.

Given a $N \in \mathbb{N}$, define a project map $\pi_{N}: H \rightarrow H$ as follows: for any $x \in H$ with $x=\sum_{k \in \mathbb{Z}^{2} \backslash\{0\}} x_{k} e_{k}$, define

$$
\pi_{N} x=\sum_{|k| \leq N} x_{k} e_{k} .
$$

We split the space $H$ into the low and high frequency parts as

$$
H=\pi_{N} H+\left(I d-\pi_{N}\right) H
$$

where $I d$ is the identity map. For the generic $N \in \mathbb{N}$ we write

$$
H^{\ell}:=\pi_{N} H, \quad H^{h}:=\left(I d-\pi_{N}\right) H .
$$


For any $x \in H, x^{\ell}:=\pi_{N} x$ and $x^{h}:=\left(I d-\pi_{N}\right) x$. It is clear that for any $\alpha>0$ one has

$$
\left|A^{\alpha} x^{\complement}\right| \leq N^{2 \alpha}\left|x^{\complement}\right|, \quad\left|A^{\alpha} x^{\complement}\right| \geq N^{2 \alpha}\left|x^{\complement}\right| .
$$

Assumption 3.1 (Highly degenerate but essentially elliptic noises assumption). There exists some fixed $N_{0} \in \mathbb{N}$ such that $\operatorname{Ran}(Q)=H^{l}:=\pi_{N_{0}} H$ and $Q x=0$ for any $x \in H^{h}$.

Remark 3.2. From this assumption, we clearly have $\operatorname{tr}\left(Q Q^{*}\right)<\infty$ and that the operator $Q: H^{l} \rightarrow H^{\ell}$ is invertible, i.e. there exists some $C_{0}>0$ such that

$$
\left|Q^{-1} x\right| \leq C_{0}|x|
$$

for any $x \in H^{l}$. In our proof, we shall choose some large (but fixed) $N_{0}$ to make the noises $Q d W_{t}$ have essential ellipticity effect (see section 5.4 of [14]).

Let us now write Theorem 1.5 in an exact form as the following Theorem 3.3 , which will be proven in next section.

Theorem 3.3. There exist some $C=C(|x|,|y|)>0$ and $\tilde{C}=\tilde{C}(|x|,|y|)>0$ such that as $\nu N_{0}^{2}>\frac{1}{2} \operatorname{tr}\left(Q Q^{*}\right)$ and $\nu>\max \left\{\operatorname{tr}\left(Q Q^{*}\right), C_{2}\right\}$ with $C_{2}>0$ defined in (3.16) below, for any bounded differentiable function $f \geq 1$ we have

$$
\begin{aligned}
P_{t} \log f(y) \leq & \log P_{t} f(x)+C\left(|x-y|^{2}+|x-y|^{4}\right) \\
& +e^{-\left(\nu N_{0}^{2}-\frac{1}{2} \operatorname{tr}\left(Q Q^{*}\right)\right) t} \tilde{C}|x-y||| D \log f \|_{\infty} .
\end{aligned}
$$

The exact values of $C$ and $\tilde{C}$ can be easily figured out from the proof. Moreover,

$$
\left|D P_{t} f(x)\right| \leq\|f\|^{2}+C+e^{-\left(\nu N_{0}^{2}-\frac{1}{2} \operatorname{tr}\left(Q Q^{*}\right)\right) t} \tilde{C}|| D \log f \|_{\infty} .
$$

3.2. Proof of Theorem 3.3. We shall apply asymptotic coupling method in the spirit of the idea in [14, Proposition 4.11]. For the more application of this method, we refer to [8], [12], [20] and [16].

Our application of the asymptotic coupling method is sketched as follows. Give any $v \in L_{l o c}^{2}([0, \infty) ; H)$ adapted to $\mathcal{F}_{t}:=\sigma\left(W_{s} ; 0 \leq s \leq t\right)$, define

$$
\tilde{W}_{t}=W_{t}+\int_{0}^{t} v_{s} d s
$$

by Girsanov theorem, we have a new probability measure $\tilde{P}$ under which $\tilde{W}$ is a Brownian motion. This probability $\tilde{P}$ is uniquely determined by

$$
\left.\frac{d \tilde{P}}{d P}\right|_{\mathcal{F}_{t}}=\exp \left\{-\int_{0}^{t} v_{s} d W_{s}-\frac{1}{2} \int_{0}^{t}\left|v_{s}\right|^{2} d s\right\} \text {. }
$$


Consider the SPDE

$$
\left\{\begin{array}{l}
d Y(t)+[A Y(t)+B(Y(t))] d t=Q d \tilde{W}_{t}, \\
Y(0)=y
\end{array}\right.
$$

Denote $Z(t)=Y(t)-X(t)$, it is easy to see that

$$
\left\{\begin{array}{l}
\partial_{t} Z(t)+A Z(t)+B(Z(t))+\tilde{B}(Z(t), X(t))=Q v_{t}, \\
Z(0)=z .
\end{array}\right.
$$

where $\tilde{B}(x, y)=B(x, y)+B(y, x)$ and $z=y-x$.

Eq. (3.8) can be split into two pieces, i.e. low frequency and high frequency dynamics as the following

$$
\partial_{t} Z^{l}(t)+A Z^{l}(t)+B^{l}(Z(t))+\tilde{B}^{l}(Z(t), X(t))=Q v_{t}
$$

with $Z^{l}(0)=z^{l}$, and

$$
\partial_{t} Z^{h}(t)+A Z^{h}(t)+B^{h}(Z(t))+\tilde{B}^{h}(Z(t), X(t))=0
$$

with $Z^{h}(0)=z^{h}$.

Let us choose the $v$ in the following way. First of all, let

$$
\left\{\begin{array}{l}
Z^{l}(t)=(1-t) z^{l} \quad 0 \leq t \leq 1 \\
Z^{l}(t)=0 \quad t>1
\end{array}\right.
$$

Plugging this $Z^{l}(t)$ into (3.10), we obtain the following PDE with unknown $Z^{h}$

$$
\left\{\begin{array}{l}
\partial_{t} Z^{h}(t)+A Z^{h}(t)+B^{h}\left(Z^{l}(t)+Z^{h}(t)\right)+\tilde{B}^{h}\left(Z^{l}(t)+Z^{h}(t), X(t)\right)=0, \\
Z^{h}(0)=z^{h},
\end{array}\right.
$$

which has a unique solution by the same method as in [24, Theorem 3.2].

Now $Z(t)=Z^{l}(t)+Z^{h}(t)$ is known. From Eq. (3.9), we have

$$
v_{t}=\left\{\begin{array}{l}
Q^{-1}\left[-z^{l}+(1-t) A z^{l}+B^{l}(Z(t))+\tilde{B}^{\iota}(Z(t), X(t))\right] \quad 0 \leq t<1 \\
Q^{-1}\left[B^{l}\left(Z^{h}(t)\right)+\tilde{B}^{l}\left(Z^{h}(t), X(t)\right)\right] \quad t \geq 1
\end{array}\right.
$$

By the relation $Z=Y-X$, we also have

$$
v_{t}=\left\{\begin{array}{l}
Q^{-1}\left[-z^{\iota}+(1-t) A z^{\iota}-B^{\iota}(Z(t))+\tilde{B}^{\iota}(Z(t), Y(t))\right] \quad 0 \leq t<1 \\
Q^{-1}\left[-B^{\iota}\left(Z^{h}(t)\right)+\tilde{B}^{\iota}\left(Z^{h}(t), Y(t)\right)\right] \quad t \geq 1
\end{array}\right.
$$

To prove Theorem 3.3, we need the following auxiliary lemmas which will be proven in the next section. 
Lemma 3.4. Let $f \geq 0$ with $\mathbb{E} f>0$. Then for any measurable function $g$, we have

$$
\mathbb{E}[f g] \leq \mathbb{E} f \log \mathbb{E} e^{g}+\mathbb{E}[f \log f]-\mathbb{E} f \log \mathbb{E} f
$$

Lemma 3.5. As $\nu>2 \operatorname{tr}\left(Q Q^{*}\right)$, we have

$$
\begin{aligned}
& \mathbb{E}_{P} \exp \left(|X(t)|^{2}+\nu \int_{0}^{t}\left|A^{\frac{1}{2}} X(s)\right|^{2} d s\right) \leq e^{|x|^{2}+\operatorname{tr}\left(Q Q^{*}\right) t} . \\
& \mathbb{E}_{\tilde{P}} \exp \left(|Y(t)|^{2}+\nu \int_{0}^{t}\left|A^{\frac{1}{2}} Y(s)\right|^{2} d s\right) \leq e^{|y|^{2}+\operatorname{tr}\left(Q Q^{*}\right) t} .
\end{aligned}
$$

Lemma 3.6. We have

$$
\langle x, B(y, x)\rangle=0, \quad\langle x, B(y, z)\rangle=-\langle z, B(y, x)\rangle
$$

for all $x, y \in D\left(A^{\frac{1}{2}}\right)$ and $z \in D\left(A^{\frac{1}{2}}\right)$. Furthermore, we have

$$
|\langle x, B(y, z)\rangle| \leq C_{1}|x||y|\left|A^{\frac{3}{2}} z\right|
$$

for all $x, y \in H$ and $z \in D\left(A^{\frac{3}{2}}\right)$, and

$$
|\langle x, B(y, z)\rangle| \leq C_{2}|x|^{1 / 2}\left|A^{\frac{1}{2}} x\right|^{1 / 2}|y|^{1 / 2}\left|A^{\frac{1}{2}} y\right|^{1 / 2}\left|A^{\frac{1}{2}} z\right| .
$$

for all $x, y, z \in D\left(A^{\frac{1}{2}}\right)$. The constants $C_{1}, C_{2}$ both only depend on the space dimension.

Lemma 3.7. For any $x, y \in D\left(A^{\frac{1}{2}}\right)$, we have

$$
\left|B^{l}(x, y)\right| \leq C_{1} N_{0}^{3}|x||y|
$$

where $C_{1}$ is the same as that in Lemma 3.6.

Lemma 3.8. Let $v$ be chosen as in (3.12). For any $p \geq 1$, if $\nu>\max \left\{C_{2} \sqrt{p / 2}, 2 \operatorname{tr}\left(Q Q^{*}\right)\right\}$ we have

$$
\begin{aligned}
& \mathbb{E}_{P} \sup _{0 \leq t \leq 1}\left|Z^{h}(t)\right|^{2 p} \leq K_{p} e^{|x|^{2}}|x-y|^{2 p}, \\
& \mathbb{E}_{\tilde{P}} \sup _{0 \leq t \leq 1}\left|Z^{h}(t)\right|^{2 p} \leq K_{p} e^{|y|^{2}}|x-y|^{2 p}
\end{aligned}
$$

and for $t>1$

$$
\begin{aligned}
& \mathbb{E}_{P}\left|Z^{h}(t)\right|^{2 p} \leq \exp \left\{-\left(2 \nu p N_{0}^{2}-\operatorname{tr}\left(Q Q^{*}\right)\right) t\right\} K_{p} e^{2|x|^{2}+2 \nu p N_{0}^{2}}|x-y|^{2 p}, \\
& \mathbb{E}_{\tilde{P}}\left|Z^{h}(t)\right|^{2 p} \leq \exp \left\{-\left(2 \nu p N_{0}^{2}-\operatorname{tr}\left(Q Q^{*}\right)\right) t\right\} K_{p} e^{2|y|^{2}+2 \nu p N_{0}^{2}}|x-y|^{2 p},
\end{aligned}
$$

where

$$
\begin{aligned}
K_{p}= & 2^{p-1} \exp \left\{C_{1} p N_{0}^{2}\left(|x-y|^{2}+|x-y|\right)+\frac{C_{1} p N_{0}^{3}}{2}+\operatorname{tr}\left(Q Q^{*}\right)\right\} \\
& \times\left[\left(1+C_{1} N_{0}^{3}+\frac{\nu N_{0}^{2}}{4}\right)^{p}+p !\left(\frac{C_{2}^{2}}{4 \nu}+\frac{C_{1} N_{0}^{3}}{2}\right)^{p}\left(\frac{C_{2}^{2} p}{4 \nu}\right)^{-p}\right]
\end{aligned}
$$


and $C_{1}, C_{2}$ are the same as in Lemma 3.6 .

Proof of Theorem 3.3. The second inequality in the theorem follows from Corollary 1.4 immediately. Let us prove the first inequality in the following two steps.

Step 1. Recalling Eq. (3.7), for any bounded differentiable function $f \geq 1$ one has

$$
P_{t} \log f(y)=\mathbb{E}_{\tilde{P}} \log f(Y(t))=\mathbb{E}_{\tilde{P}} \log f\left(Y^{l}(t)+Y^{h}(t)\right) .
$$

By (3.11) we have $Z^{l}(t)=0$, i.e. $X^{l}(t)=Y^{l}(t)$ for all $t \geq 1$, hence

$$
P_{t} \log f(y)=\mathbb{E}_{\tilde{P}}\left[\log f\left(X^{l}(t)+Y^{h}(t)\right)\right], \quad t \geq 1 .
$$

Writing $z=x-y$, by Lemmas 3.8 and 3.4 we have

$$
\begin{aligned}
P_{t} \log f(y) & =\mathbb{E}_{\tilde{P}}\left[\log f\left(X^{l}(t)+Y^{h}(t)\right)-\log f\left(X^{l}(t)+X^{h}(t)\right)\right]+\mathbb{E}_{\tilde{P}} \log f(X(t)) \\
& \leq\|D \log f\|_{\infty}\left\{\mathbb{E}_{\tilde{P}}\left|Z^{h}(t)\right|^{2}\right\}^{\frac{1}{2}}+\mathbb{E}_{P}\left[\frac{d \tilde{P}}{d P} \log f(X(t))\right] \\
& \leq \exp \left\{-\left(\nu N_{0}^{2}-\frac{1}{2} \operatorname{tr}\left(Q Q^{*}\right)\right) t+|y|^{2}+\nu N_{0}^{2}\right\} \sqrt{K_{1}}|z|\|D \log f\|_{\infty} \\
& +\mathbb{E}_{P}\left[\frac{d \tilde{P}}{d P} \log \frac{d \tilde{P}}{d P}\right]+\log \mathbb{E}_{P} f(X(t))
\end{aligned}
$$

where $K_{1}$ is defined in Lemma 3.8. For the entropy term above, by (3.5) and (3.6),

$$
\begin{aligned}
\mathbb{E}_{P}\left[\frac{d \tilde{P}}{d P} \log \frac{d \tilde{P}}{d P}\right] & =\mathbb{E}_{\tilde{P}}\left[-\int_{0}^{t} v_{s} d W_{s}-\frac{1}{2} \int_{0}^{t}\left|v_{s}\right|^{2} d s\right] \\
& =\mathbb{E}_{\tilde{P}}\left[-\int_{0}^{t} v_{s} d \tilde{W}_{s}+\frac{1}{2} \int_{0}^{t}\left|v_{s}\right|^{2} d s\right]=\frac{1}{2} \mathbb{E}_{\tilde{P}} \int_{0}^{t}\left|v_{s}\right|^{2} d s .
\end{aligned}
$$

We claim

$$
\mathbb{E}_{\tilde{P}} \int_{0}^{t}\left|v_{s}\right|^{2} d s \leq\left(L_{1}+L_{3}\right)|z|^{4}+\left(L_{2}+L_{4}\right)|z|^{2} .
$$

where $L_{1} \cdots, L_{4}$ are defined in Step 2 below. From (3.20), (3.21) and (3.22), we have

$$
\begin{aligned}
P_{t} \log f(y) & \leq \log P_{t} f(x)+\left(L_{1}+L_{3}\right)|z|^{4}+\left(L_{2}+L_{4}\right)|z|^{2} \\
& +\exp \left\{-\left(\nu N_{0}^{2}-\frac{1}{2} \operatorname{tr}\left(Q Q^{*}\right)\right) t+|y|^{2}+\nu p N_{0}^{2}\right\} \sqrt{K_{1}}|z||| D \log f \|_{\infty} .
\end{aligned}
$$

By the definitions of $K_{1}, L_{1}, \cdots L_{4}$ and recalling $z=y-x$, we conclude the proof up to proving (3.22), and can easily figure out the exactly values of $C$ and $\tilde{C}$ in 
the theorem.

Step 2. Let us prove (3.22). We first consider $\mathbb{E}_{\tilde{P}} \int_{0}^{1}\left|v_{s}\right|^{2} d s$. By (3.4), (3.13) and $\left|A z^{\complement}\right| \leq N_{0}^{2}|z|$, one has

$$
\mathbb{E}_{\tilde{P}} \int_{0}^{1}\left|v_{s}\right|^{2} d s \leq 3 C_{0}^{2}\left(4 N_{0}^{4}|z|^{2}+\mathbb{E}_{\tilde{P}} \int_{0}^{1}\left|B^{l}(Z(s))\right|^{2}+\left|\tilde{B}^{c}(Z(s), Y(s))\right|^{2} d s\right)
$$

By Lemma 3.7, (3.18) and $\left|Z^{l}(t)\right| \leq|z|$ for $0 \leq t \leq 1$, we have

$$
\begin{aligned}
& \mathbb{E}_{\tilde{P}} \int_{0}^{1}\left|B^{l}(Z(s))\right|^{2} d s \leq C_{1}^{2} N_{0}^{6} \int_{0}^{1} \mathbb{E}_{\tilde{P}}|Z(s)|^{4} d s \\
& =8 C_{1}^{2} N_{0}^{6} \int_{0}^{1} \mathbb{E}_{\tilde{P}}\left(\left|Z^{l}(s)\right|^{4}+\left|Z^{h}(s)\right|^{4}\right) d s \\
& \leq 8 C_{1}^{2} N_{0}^{6}\left(1+K_{2} e^{|y|^{2}}\right)|z|^{4} .
\end{aligned}
$$

Moreover, by Lemmas 3.7 and 3.5, (3.18) and a similar argument as the above, one has

$$
\begin{aligned}
& \int_{0}^{1}\left|\tilde{B}^{l}(Z(s), Y(s))\right|^{2} d s \leq 4 C_{1}^{2} N_{0}^{6} \int_{0}^{1} \mathbb{E}_{\tilde{P}}|Z(s)|^{2}|Y(s)|^{2} d s \\
& \leq 4 C_{1}^{2} N_{0}^{6} \int_{0}^{1}\left(\mathbb{E}_{\tilde{P}} Z^{4}(s)\right)^{\frac{1}{2}}\left(\mathbb{E}_{\tilde{P}}|Y(s)|^{4}\right)^{\frac{1}{2}} d s \\
& \leq 4 C_{1}^{2} N_{0}^{6} \int_{0}^{1}\left(\mathbb{E}_{\tilde{P}} Z^{4}(s)\right)^{\frac{1}{2}}\left(2 \mathbb{E}_{\tilde{P}} e^{|Y(s)|^{2}}\right)^{\frac{1}{2}} d s \\
& \leq 4 \sqrt{2} C_{1}^{2} N_{0}^{6} \sqrt{1+K_{2} e^{|y|^{2}}} \exp \left\{\frac{1}{2}|y|^{2}+\frac{1}{2} \operatorname{tr}\left(Q Q^{*}\right)\right\}|z|^{2} .
\end{aligned}
$$

Collecting all the above, we have

$$
\mathbb{E}_{\tilde{P}} \int_{0}^{1}\left|v_{s}\right|^{2} d s \leq L_{1}|z|^{4}+L_{2}|z|^{2} .
$$

where

$$
\begin{gathered}
L_{1}=24 C_{0}^{2} C_{1}^{2} N_{0}^{6}\left(1+K_{2} e^{|y|^{2}}\right) \\
L_{2}=3 C_{0}^{2}\left(4 N_{0}^{4}+4 \sqrt{2} C_{1}^{2} N_{0}^{6} \sqrt{1+K_{2} e^{|y|^{2}}} e^{\left[|y|^{2}+\operatorname{tr}\left(Q Q^{*}\right)\right] / 2}\right) .
\end{gathered}
$$

Now let us estimate $\mathbb{E}_{\tilde{P}} \int_{1}^{t}\left|v_{s}\right|^{2} d s$, by (3.13) one has

$$
\mathbb{E}_{\tilde{P}} \int_{1}^{t}\left|v_{s}\right|^{2} d s \leq 2 C_{0}^{2} \mathbb{E}_{\tilde{P}} \int_{1}^{t}\left(\left|B^{l}\left(Z^{h}(s)\right)\right|^{2}+\left|\tilde{B}^{\iota}\left(Z^{h}(s), Y(s)\right)\right|^{2}\right) d s .
$$


By a similar argument as for proving (3.23) and thanks to (3.19), when $\nu N_{0}^{2}>$ $\frac{1}{2} \operatorname{tr}\left(Q Q^{*}\right)$ one has

$$
\mathbb{E}_{\tilde{P}} \int_{1}^{t}\left|B^{l}\left(Z^{h}(s)\right)\right|^{2} d s \leq C_{1}^{2} N_{0}^{6} \int_{1}^{t} \mathbb{E}_{\tilde{P}}\left|Z^{h}(s)\right|^{4} d s \leq \frac{C_{1}^{2} N_{0}^{6} e^{2|y|^{2}+4 \nu N_{0}^{2}} K_{2}}{4 \nu N_{0}^{2}-\operatorname{tr}\left(Q Q^{*}\right)}|z|^{4},
$$

and

$$
\begin{aligned}
& \mathbb{E}_{\tilde{P}} \int_{1}^{t}\left|\tilde{B}^{l}\left(Z^{h}(s), Y(s)\right)\right|^{2} d s \leq 2 C_{1}^{2} N_{0}^{6} \int_{1}^{t} \mathbb{E}_{\tilde{P}}\left|Z^{h}(s)\right|^{2}|Y(s)|^{2} d s \\
\leq & 2 C_{1}^{2} N_{0}^{6} \int_{1}^{t}\left(\mathbb{E}_{\tilde{P}}\left|Z^{h}(s)\right|^{4}\right)^{1 / 2}\left(\mathbb{E}_{\tilde{P}}|Y(s)|^{4}\right)^{1 / 2} d s \leq \frac{2 C_{1}^{2} N_{0}^{6} \sqrt{2 K_{2}} e^{\frac{3}{2}}|y|^{2}+2 \nu N_{0}^{2}}{2 \nu N_{0}^{2}-\operatorname{tr}\left(Q Q^{*}\right)}|z|^{2}
\end{aligned}
$$

Therefore,

$$
\mathbb{E}_{\tilde{P}} \int_{1}^{t}\left|v_{s}\right|^{2} d s \leq L_{3}|z|^{4}+L_{4}|z|^{2}
$$

where

$$
L_{3}=\frac{2 C_{0}^{2} C_{1}^{2} N_{0}^{6} e^{2|y|^{2}+4 \nu N_{0}^{2}} K_{2}}{4 \nu N_{0}^{2}-\operatorname{tr}\left(Q Q^{*}\right)}, \quad L_{4}=\frac{4 C_{0}^{2} C_{1}^{2} N_{0}^{6} \sqrt{2 K_{2}} e^{\frac{3}{2}|y|^{2}+2 \nu N_{0}^{2}}}{2 \nu N_{0}^{2}-\operatorname{tr}\left(Q Q^{*}\right)} .
$$

\section{Proof of aUxiliary LEMmas in SECTion 3}

Some of the first four lemmas are well known. Since their proofs are short, it is very convenient to repeat them here.

Proof of Lemma 3.4. Since all the expectations restricted on the set $\{x: f(x)=0\}$ are zero, without loss of generality, we can assume that $f>0$ a.e.. We can also simply assume that $\mathbb{E} f=1$, otherwise one can replace $f$ by $\frac{f}{\mathbb{E} f}$. We have

$$
\begin{aligned}
\mathbb{E}[f g] \leq \mathbb{E}\left[f \log e^{g}\right] & =\mathbb{E}\left[f \log \frac{e^{g}}{f}\right]+\mathbb{E}[f \log f] \\
& \leq \log \mathbb{E}\left[f \frac{e^{g}}{f}\right]+\mathbb{E}[f \log f]=\mathbb{E} e^{g}+\mathbb{E}[f \log f] .
\end{aligned}
$$

Proof of Lemma 3.5. The proofs for the two claims are the same, so we only prove the first one. By Itô formula, we have

$$
\begin{aligned}
& |X(t)|^{2}+\nu \int_{0}^{t}\left|A^{\frac{1}{2}} X(s)\right|^{2} d s \\
& =|x|^{2}+\operatorname{tr}\left(Q Q^{*}\right) t+2 \int_{0}^{t}\left\langle X(s), Q d W_{s}\right\rangle-\nu \int_{0}^{t}\left|A^{\frac{1}{2}} X(s)\right|^{2} d s .
\end{aligned}
$$


By $|x| \leq\left|A^{\frac{1}{2}} x\right|$ and $\left|Q^{*} x\right|^{2} \leq \operatorname{tr}\left(Q Q^{*}\right)|x|^{2}$, we have

$$
\begin{aligned}
& \mathbb{E}_{P} \exp \left\{2 \int_{0}^{t}\left\langle X(s), Q d W_{s}\right\rangle-\nu \int_{0}^{t}\left|A^{\frac{1}{2}} X(s)\right|^{2} d s\right\} \\
& \leq \mathbb{E}_{P} \exp \left\{2 \int_{0}^{t}\left\langle X(s), Q d W_{s}\right\rangle-2 \int_{0}^{t}\left|Q^{*} X(s)\right|^{2} d s+\left(2 \operatorname{tr}\left(Q Q^{*}\right)-\nu\right) \int_{0}^{t}|X(s)|^{2} d s\right\} .
\end{aligned}
$$

Since $\exp \left\{2 \int_{0}^{t}\left\langle X(s), Q d W_{s}\right\rangle-2 \int_{0}^{t}\left|Q^{*} X(s)\right|^{2} d s\right\}$ is a martingale, as $\nu>2 \operatorname{tr}\left(Q Q^{*}\right)$, one has

$$
\mathbb{E}_{P} \exp \left\{|X(t)|^{2}+\nu \int_{0}^{t}\left|A^{\frac{1}{2}} X(s)\right|^{2} d s\right\} \leq e^{|x|^{2}+\operatorname{tr}\left(Q Q^{*}\right) t}
$$

Proof of Lemma 3.6. (3.14) is classical, one can, for instance, refers to [24]. We clearly have

$$
|\langle x, B(y, z)\rangle| \leq|x||y||| \nabla z \|_{\infty} \leq C_{1}|x||y|\left|A^{\frac{3}{2}} z\right|
$$

since $z=\sum_{k \in \mathbb{Z}^{2} \backslash\{0\}} z_{k} e^{i 2 \pi k \cdot x}$ and hence

$$
\|\nabla z\|_{\infty} \leq C \sum_{k \in \mathbb{Z}^{2} \backslash\{0\}}|k|\left|z_{k}\right| \leq C \sqrt{\sum_{k \in \mathbb{Z}^{2} \backslash\{0\}}|k|^{-4}} \sqrt{\sum_{k \in \mathbb{Z}^{2} \backslash\{0\}}|k|^{6}\left|z_{k}\right|^{2}}
$$

As for (3.16), by Hölder's inequality, the classical Sobolev embedding $\|y\|_{L^{4}} \leq$ $C\left|A^{\frac{1}{4}} y\right|\left(\left[13\right.\right.$, Theorem 6.16 and Remark 6.17]) and the easy interpolation $\left|A^{\frac{1}{4}} x\right| \leq$ $|x|^{1 / 2}\left|A^{\frac{1}{2}} x\right|^{1 / 2}$, we have

$$
|\langle x, B(y, z)\rangle| \leq\|x\|_{L^{4}}|| y \|_{L^{4}}\left|A^{\frac{1}{2}} z\right| \leq C_{2}|x|^{1 / 2}\left|A^{\frac{1}{2}} x\right|^{1 / 2}|y|^{1 / 2}\left|A^{\frac{1}{2}} y\right|^{1 / 2}\left|A^{\frac{1}{2}} z\right| .
$$

Proof of Lemma 3.7. For any $z \in H$ with $z^{h}=0$, it is clear from the first inequality of (3.3) that

$$
\left|A^{\frac{3}{2}} z\right| \leq N_{0}^{3}|z|
$$

By (3.14) and (3.15), one has

$$
\left|\left\langle z, B^{l}(x, y)\right\rangle\right|=|\langle y, B(x, z)\rangle| \leq C_{1}\left|A^{\frac{3}{2}} z\right||y||x| \leq C_{1} N_{0}^{3}|y||x||z|
$$

Proof of Lemma 3.8. The proof of the claims for $P$ and $\tilde{P}$ are the same, so we only show that for the former. We split the proof into the following three steps.

Step 1. By (3.10) we have

$$
\partial_{t}\left|Z^{h}(t)\right|^{2}+2 \nu\left|A^{\frac{1}{2}} Z^{h}(t)\right|^{2}=-2\left\langle Z^{h}(t), B^{h}(Z(t))\right\rangle-2\left\langle Z^{h}(t), \tilde{B}^{h}(Z(t), X(t))\right\rangle .
$$


By (3.14), (3.15) and (3.3) we have

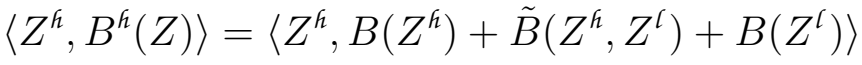

$$
\begin{aligned}
& =\left\langle Z^{h}, B\left(Z^{h}, Z^{\natural}\right)\right\rangle+\left\langle Z^{h}, B\left(Z^{\natural}\right)\right\rangle \\
& \leq C_{1} N_{0}^{3}\left(\left|Z^{\complement}\right|\left|Z^{\complement}\right|^{2}+\left|Z^{\complement}\right|^{2}\left|Z^{\complement}\right|\right) \text {. }
\end{aligned}
$$

As for the second term on the r.h.s. of (4.1), we have by (3.16)

$$
\left|\left\langle Z^{h}, B(Z, X)\right\rangle\right| \leq C_{2}\left|Z^{h}\right|^{1 / 2}\left|A^{\frac{1}{2}} Z^{h}\right|^{1 / 2}|Z|^{1 / 2}\left|A^{\frac{1}{2}} Z\right|^{1 / 2}\left|A^{\frac{1}{2}} X\right|,
$$

and have by (3.14), (3.15) and (3.3)

$$
\left|\left\langle Z^{h}, B(X, Z)\right\rangle\right|=\left|\left\langle Z^{h}, B\left(X, Z^{\complement}\right)\right\rangle\right| \leq C_{1} N_{0}^{3}\left|Z^{h}\right||X|\left|Z^{\complement}\right| \text {. }
$$

Step 2. Let us now estimate $\mathbb{E} \sup _{0<t<1}\left|Z^{h}(t)\right|^{2 p}$. By (4.2), Cauchy inequality and $\left|Z^{l}(t)\right| \leq|z|$ for $0 \leq t \leq 1$ (see (3.11) $)$ one has

$$
\begin{aligned}
\left|\left\langle Z^{h}, B^{h}(Z)\right\rangle\right| & \leq C_{1} N_{0}^{3}|z|\left|Z^{h}\right|^{2}+\frac{1}{2} C_{1} N_{0}^{3}|z|^{2}\left|Z^{h}\right|^{2}+\frac{1}{2} C_{1} N_{0}^{3}|z|^{2} \\
& \leq C_{1} N_{0}^{3}\left(|z|^{2}+|z|\right)\left|Z^{h}\right|^{2}+C_{1} N_{0}^{3}|z|^{2} .
\end{aligned}
$$

Applying Young's inequality (two times), (4.3) and $\left|A^{\frac{1}{2}} z^{\complement}\right| \leq N_{0}|z|$, we have

$$
\begin{aligned}
\left|\left\langle Z^{h}, B(Z, X)\right\rangle\right| & \leq \frac{\nu}{2}\left|A^{\frac{1}{2}} Z^{h}\right|\left|A^{\frac{1}{2}} Z\right|+\frac{C_{2}^{2}}{4 \nu}\left|A^{\frac{1}{2}} X\right|^{2}\left|Z^{h}\right|^{2}+\frac{C_{2}^{2}}{4 \nu}\left|A^{\frac{1}{2}} X\right|^{2}|Z|^{2} \\
& \leq \frac{3 \nu}{4}\left|A^{\frac{1}{2}} Z^{h}\right|^{2}+\frac{\nu}{4}\left|A^{\frac{1}{2}} Z^{\complement}\right|^{2}+\frac{C_{2}^{2}}{4 \nu}\left|A^{\frac{1}{2}} X\right|^{2}\left|Z^{\complement}\right|^{2}+\frac{C_{2}^{2}}{4 \nu}\left|A^{\frac{1}{2}} X\right|^{2}\left|Z^{\complement}\right|^{2} \\
& \leq \frac{3 \nu}{4}\left|A^{\frac{1}{2}} Z^{h}\right|^{2}+\frac{\nu N_{0}^{2}}{4}|z|^{2}+\frac{C_{2}^{2}}{4 \nu}\left|A^{\frac{1}{2}} X\right|^{2}\left|Z^{h}\right|^{2}+\frac{C_{2}^{2}}{4 \nu}\left|A^{\frac{1}{2}} X\right|^{2}|z|^{2} .
\end{aligned}
$$

By (4.4), $|X| \leq\left|A^{\frac{1}{2}} X\right|$ and $\left|Z^{l}(t)\right| \leq\left|z^{\complement}\right|$ for $0 \leq t \leq 1$, we get

$$
\left|\left\langle Z^{h}, B(X, Z)\right\rangle\right| \leq \frac{C_{1} N_{0}^{3}}{2}\left|Z^{\hbar}\right|^{2}+\frac{C_{1} N_{0}^{3}}{2}\left|A^{\frac{1}{2}} X\right|^{2}|z|^{2} .
$$

The above three inequalities and (4.1) imply

$$
\begin{aligned}
\partial_{t}\left|Z^{h}(t)\right|^{2} \leq & {\left[C_{1} N_{0}^{2}\left(|z|^{2}+|z|\right)+\frac{C_{2}^{2}}{4 \nu}\left|A^{\frac{1}{2}} X\right|^{2}+\frac{C_{1} N_{0}^{3}}{2}\right]\left|Z^{h}(t)\right|^{2} } \\
& +\left[C_{1} N_{0}^{3}|z|^{2}+\frac{\nu N_{0}^{2}}{4}|z|^{2}+\frac{C_{2}^{2}}{4 \nu}\left|A^{\frac{1}{2}} X\right|^{2}|z|^{2}+\frac{C_{1} N_{0}^{3}}{2}\left|A^{\frac{1}{2}} X\right|^{2}|z|^{2}\right],
\end{aligned}
$$

thus for any $0 \leq t \leq 1$

$$
\begin{aligned}
\left|Z^{h}(t)\right|^{2} & \leq \exp \left\{C_{1} N_{0}^{2}\left(|z|^{2}+|z|\right)+\frac{C_{1} N_{0}^{3}}{2}+\frac{C_{2}^{2}}{4 \nu} \int_{0}^{1}\left|A^{\frac{1}{2}} X(s)\right|^{2} d s\right\} \\
& \times\left[\left|z^{h}\right|^{2}+C_{1} N_{0}^{3}|z|^{2}+\frac{\nu N_{0}^{2}}{4}|z|^{2}+\left(\frac{C_{2}^{2}}{4 \nu}+\frac{C_{1} N_{0}^{3}}{2}\right)|z|^{2} \int_{0}^{1}\left|A^{\frac{1}{2}} X(s)\right|^{2} d s\right] .
\end{aligned}
$$


It follows that

$$
\begin{aligned}
\sup _{0 \leq t \leq 1}\left|Z^{h}(t)\right|^{2 p} & \leq 2^{p-1} \exp \left\{C_{1} p N_{0}^{2}\left(|z|^{2}+|z|\right)+\frac{C_{1} p N_{0}^{3}}{2}\right\} \exp \left\{\frac{C_{2}^{2} p}{4 \nu} \int_{0}^{1}\left|A^{\frac{1}{2}} X(s)\right|^{2} d s\right\} \\
& \times\left[\left(1+C_{1} N_{0}^{3}+\frac{\nu N_{0}^{2}}{4}\right)^{p}+\left(\frac{C_{2}^{2}}{4 \nu}+\frac{C_{1} N_{0}^{3}}{2}\right)^{p}\left(\int_{0}^{1}\left|A^{\frac{1}{2}} X(s)\right|^{2} d s\right)^{p}\right]|z|^{2 p} .
\end{aligned}
$$

By Lemma 3.5, as $\nu>\max \left\{C_{2} \sqrt{p} / 2,2 \operatorname{tr}\left(Q Q^{*}\right)\right\}$,

$$
\mathbb{E} \exp \left\{\frac{C_{2}^{2} p}{4 \nu} \int_{0}^{1}\left|A^{\frac{1}{2}} X(s)\right|^{2} d s\right\} \leq e^{|x|^{2}+\operatorname{tr}\left(Q Q^{*}\right)}
$$

and

$$
\begin{aligned}
& \mathbb{E}\left[\exp \left\{\frac{C_{2}^{2} p}{4 \nu} \int_{0}^{1}\left|A^{\frac{1}{2}} X(s)\right|^{2} d s\right\}\left(\int_{0}^{1}\left|A^{\frac{1}{2}} X(s)\right|^{2} d s\right)^{p}\right] \\
& \leq p !\left(\frac{C_{2}^{2} p}{4 \nu}\right)^{-p} \mathbb{E} \exp \left\{\frac{C_{2}^{2} p}{4 \nu} \int_{0}^{1}\left|A^{\frac{1}{2}} X(s)\right|^{2} d s\right\} \leq p !\left(\frac{C_{2}^{2} p}{4 \nu}\right)^{-p} e^{|x|^{2}+\operatorname{tr}\left(Q Q^{*}\right)} .
\end{aligned}
$$

Collecting the above three inequalities, we get

$$
\begin{aligned}
\mathbb{E} \sup _{0 \leq t \leq 1}\left|Z^{h}(t)\right|^{2 p} & \leq 2^{p-1} \exp \left\{C_{1} p N_{0}^{2}\left(|z|^{2}+|z|\right)+\frac{C_{1} p N_{0}^{3}}{2}\right\} \\
& \times\left[\left(1+C_{1} N_{0}^{3}+\frac{\nu N_{0}^{2}}{4}\right)^{p}+p !\left(\frac{C_{2}^{2}}{4 \nu}+\frac{C_{1} N_{0}^{3}}{2}\right)^{p}\left(\frac{C_{2}^{2} p}{4 \nu}\right)^{-p}\right] e^{|x|^{2}+\operatorname{tr}\left(Q Q^{*}\right)}|z|^{2 p}
\end{aligned}
$$

Step 3. As $t>1, Z^{l}(t)=0$ by (3.11). From (4.1)-(4.4),

$$
\partial_{t}\left|Z^{\hbar}\right|^{2}+2 \nu\left|A^{\frac{1}{2}} Z^{\hbar}\right|^{2} \leq C_{2}\left|Z^{\hbar}\right|\left|A^{\frac{1}{2}} Z^{\hbar}\right|\left|A^{\frac{1}{2}} X\right|,
$$

which, together with Young's inequality, implies

$$
\partial_{t}\left|Z^{h}\right|^{2}+\nu\left|A^{\frac{1}{2}} Z^{h}\right|^{2} \leq \frac{C_{2}^{2}}{4 \nu}\left|A^{\frac{1}{2}} X\right|^{2}\left|Z^{h}\right|^{2} .
$$

By the second inequality of (3.3) we further have

$$
\partial_{t}\left|Z^{h}\right|^{2}+\nu N_{0}^{2}\left|Z^{h}\right|^{2} \leq \frac{C_{2}^{2}}{4 \nu}\left|A^{\frac{1}{2}} X\right|^{2}\left|Z^{h}\right|^{2} .
$$

Therefore, for all $t>1$

$$
\begin{aligned}
\mathbb{E}\left|Z^{h}(t)\right|^{2 p} & \leq \mathbb{E} \exp \left\{-\nu p N_{0}^{2}(t-1)+\frac{C_{2}^{2} p}{4 \nu} \int_{1}^{t}\left|A^{\frac{1}{2}} X(s)\right|^{2} d s\right\}\left|Z^{h}(1)\right|^{2 p} \\
& \leq\left(\mathbb{E} \exp \left\{-2 \nu p N_{0}^{2}(t-1)+\frac{C_{2}^{2} p}{2 \nu} \int_{1}^{t}\left|A^{\frac{1}{2}} X(s)\right|^{2} d s\right\}\right)^{1 / 2}\left(\mathbb{E}\left|Z^{h}(1)\right|^{4 p}\right)^{1 / 2} \\
& \leq \exp \left\{2 \nu p N_{0}^{2}+|x|^{2}-\left(2 \nu p N_{0}^{2}-\operatorname{tr}\left(Q Q^{*}\right)\right) t\right\}\left(\mathbb{E}\left|Z^{h}(1)\right|^{4 p}\right)^{1 / 2}
\end{aligned}
$$


as $\nu>\max \left\{C_{2} \sqrt{p / 2}, 2 \operatorname{tr}\left(Q Q^{*}\right)\right\}$, where the last inequality is due to Lemma 3.5. This, together with the last inequality in Step 2, immediately implies (3.19)

\section{REFERENCES}

1. Shigeki Aida and Hiroshi Kawabi, Short time asymptotics of a certain infinite dimensional diffusion process, Stochastic analysis and related topics, VII (Kusadasi, 1998), Progr. Probab., vol. 48, Birkhäuser Boston, Boston, MA, 2001, pp. 77-124. MR MR1915450 (2003m:60219)

2. Shigeki Aida and Tusheng Zhang, On the small time asymptotics of diffusion processes on path groups, Potential Anal. 16 (2002), no. 1, 67-78. MR MR1880348 (2003e:58052)

3. M. Arnaudon, A. Thalmaier, F.-Y. Wang, Harnack inequality and heat kernel estimates on manifolds with curvature unbounded below, Bull. Sci. Math. 130(2006) 223233.

4. M. Arnaudon, A. Thalmaier, F.-Y. Wang, Gradient estimates and Harnack inequalities on non-compact Riemannian manifolds, Stoch. Proc. Appl. 119(2009), 3653-3670.

5. S. G. Bobkov, I. Gentil, M. Ledoux, Hypercontractivity of Hamilton-Jacobi equations, J. Math. Pures Appl. 80(2001), 669-696.

6. Sergey G. Bobkov, Ivan Gentil, and Michel Ledoux, Hypercontractivity of HamiltonJacobi equations, J. Math. Pures Appl. (9) 80 (2001), no. 7, 669-696. MR MR1846020 (2003b:47073)

7. G. Da Prato, M. Röckner, F.-Y. Wang, Singular stochastic equations on Hilbert spaces: Harnack inequalities for their transition semigroups, J. Funct. Anal. 257(2009), 9921017.

8. Weinan E, J. C. Mattingly, and Ya. Sinai, Gibbsian dynamics and ergodicity for the stochastically forced Navier-Stokes equation, Comm. Math. Phys. 224 (2001), no. 1, 83-106, Dedicated to Joel L. Lebowitz. MR 1868992 (2002m:76024)

9. A. Es-Sarhir, M.-K. V. Renesse and M. Scheutzow, Harnack inequality for functional SDEs with bounded memory, Electron. Commun. Probab. 14 (2009), 560-565.

10. Franco Flandoli and Bohdan Maslowski, Ergodicity of the 2-D Navier-Stokes equation under random perturbations, Comm. Math. Phys. 172 (1995), no. 1, 119-141. MR 1346374 (96g:35223)

11. Fu-Zhou Gong and Feng-Yu Wang, Heat kernel estimates with application to compactness of manifolds, Q. J. Math. 52 (2001), no. 2, 171-180. MR MR1838361 (2002c:58039)

12. M. Hairer, Exponential mixing properties of stochastic PDEs through asymptotic coupling, Probab. Theory Related Fields 124 (2002), no. 3, 345-380. MR 1939651 (2004j:60135)

13. Martin Hairer, An introduction to stochastic PDEs, 2009, http://www.hairer.org/notes/SPDEs.pdf

14. Martin Hairer and Jonathan C. Mattingly, Ergodicity of the 2D Navier-Stokes equations with degenerate stochastic forcing, Ann. of Math. (2) 164 (2006), no. 3, 993-1032. MR MR2259251 (2008a:37095)

15. _ A theory of hypoellipticity and unique ergodicity for semilinear stochastic pdes, 2008, arXiv:0808.1361v1.

16. Martin Hairer and Jonathan C. Mattingly and Michael Scheutzow, Asymptotic coupling and a weak form of Harris' theorem with applications to stochastic delay equations, 2009, arXiv:0902.4495v2.

17. Hiroshi Kawabi, The parabolic Harnack inequality for the time dependent Ginzburg-Landau type SPDE and its application, Potential Anal. 22 (2005), no. 1, 61-84. MR MR2127731 (2006j:60066)

18. S. Kuksin, A. Shirikyan, Mathematics of 2D Statistical Hydrodynamics, manuscript of a book (available on www.u-cergy.fr/shirikyan/book.html) 
19. Wei Liu and Feng-Yu Wang, Harnack inequality and strong Feller property for stochastic fast-diffusion equations, J. Math. Anal. Appl. 342 (2008), no. 1, 651-662. MR MR2440828 (2009k:60137)

20. Jonathan C. Mattingly, Exponential convergence for the stochastically forced Navier-Stokes equations and other partially dissipative dynamics, Comm. Math. Phys. 230 (2002), no. 3, 421-462. MR 1937652 (2004a:76039)

21. Michael Röckner and Feng-Yu Wang, Log-Harnack inequality for stochastic differential equations in Hilbert spaces and its consequences, Infin. Dimens. Anal. Quantum Probab. Relat. Top. 13 (2010), no. 1, 27-37. MR 2646789

22. _ Harnack and functional inequalities for generalized Mehler semigroups, J. Funct. Anal. 203 (2003), no. 1, 237-261. MR MR1996872 (2005d:47077)

23. - Supercontractivity and ultracontractivity for (non-symmetric) diffusion semigroups on manifolds, Forum Math. 15 (2003), no. 6, 893-921. MR MR2010284 (2005b:58059)

24. Roger Temam, Navier-Stokes equations and nonlinear functional analysis, second ed., CBMSNSF Regional Conference Series in Applied Mathematics, vol. 66, Society for Industrial and Applied Mathematics (SIAM), Philadelphia, PA, 1995. MR 1318914 (96e:35136)

25. Cédric Villani, Topics in optimal transportation, Graduate Studies in Mathematics, vol. 58, American Mathematical Society, Providence, RI, 2003. MR MR1964483 (2004e:90003)

26. Feng-Yu Wang, Logarithmic Sobolev inequalities on noncompact Riemannian manifolds, Probab. Theory Related Fields 109 (1997), no. 3, 417-424. MR MR1481127 (98i:58253)

27. __ Harnack inequalities for log-Sobolev functions and estimates of log-Sobolev constants, Ann. Probab. 27 (1999), no. 2, 653-663. MR MR1698947 (2000i:58067)

28. L_ Logarithmic Sobolev inequalities: conditions and counterexamples, J. Operator Theory 46 (2001), no. 1, 183-197. MR MR1862186 (2003g:58060)

29. Harnack inequality and applications for stochastic generalized porous media equations, Ann. Probab. 35 (2007), no. 4, 1333-1350. MR MR2330974 (2008e:60192)

30. F.-Y. Wang, Harnack inequalities on manifolds with boundary and applications, J. Math. Pures Appl. 94(2010), 304-321.

31. F.-Y. Wang, Harnack inequality for SDE with multiplicative noise and extension to Neumann semigroup on non-convex manifolds, to appear in Annals of Probab. arXiv:0911.1644

32. Feng-Yu Wang, Jiang-Lun Wu, and Lihu Xu, Harnack and log-harnack inequalities of stochastic burgers type equations, arXiv:1009.5948v1.

33. F.-Y. Wang, L. Xu, Derivative formula and its application to stochastic hyperdissipative Navier-Stokes/Burgers equations, arXiv:1009.1464

TU Berlin, Fakultät II, Institut für Mathematik, Str $\alpha \beta$ E Des 17 . Juni 136 , D-10623 Berlin, Germany

E-mail address: xu@math.tu-berlin.de 